\title{
Sistemas de Graduação para Carcinoma de Mama: Estudo Comparativo da Concordância Cito-Histologica
}

\author{
Grading Systems for Breast Carcinoma: \\ Comparative Study of Cytohistological Agreement
}

Karla Adriana Nascimento Dantas, Gilda da Cunha Santos, Osvaldo Giannotti Filho

\begin{abstract}
RESUM0
Objetivos: avaliar a concordância das classificações citológicas de graduação tumoral e nuclear nos esfregaços de punção aspirativa por agulha fina (PAAF) de carcinoma de mama com os métodos utilizados nos espécimes histológicos e compará-los para identificar aqueles que apresentam melhores resultados.

Métodos: a avaliação da concordância cito-histológica foi realizada em estudo retrospectivo de 50 casos de PAAF de carcinoma ductal invasivo de mama, confirmados histologicamente, aplicando-se de forma comparativa cinco sistemas de graduação. As classificações foram separadas segundo critérios de graduação tumoral (critérios nucleares e arquiteturais sistemas de Mouriquand e de Guilford) e nuclear (sistemas de Black modificado por Fisher$B M$, de Black simplificado - BS e de Hunt). As classificações utilizadas na histologia foram os sistemas de graduação de Scarff-Bloom-Richardson modificado por Elston (SBR modificado), para avaliação tumoral, e os de BM, para avaliação nuclear.

Resultados: os sistemas de graduação citológica que apresentaram maior concordância foram os sistemas de $B M(K=0,358)$ e BS $(K=0,302)$, baseados em critérios nucleares (anisonucleose, tamanho, mitose e cromatina). Dentre os sistemas de graduação citológica que apresentam critérios nucleares e arquiteturais, a classificação de Guilford demonstrou maior concordância $(K=0,260)$, possivelmente pelo número maior de variáveis utilizadas, possibilitando menor margem de erro.

Conclusão: no presente estudo, estes métodos mostraram-se regulares como sistemas de graduação citológica.
\end{abstract}

PALAVRAS-CHAVE: Carcinoma de mama. Graduação nuclear. Graduação histológica. Punção aspirativa por agulha fina.

\section{Introdução}

Dentre os tumores malignos de mama, os carcinomas ductais invasivos representam o maior grupo, constituindo cerca de 65 a $80 \%$ dos carcinomas mamários. O quadro morfológico desses

Departamento de Patologia Escola Paulista de Medicina UNIFESP (Universidade Federal de São Paulo).

Correspondência:

Gilda da Cunha Santos

Departamento de Patologia Escola Paulista de Medicina Universidade Federal de São Paulo (UNIFESP)

Rua Botucatu, 740 - Vila Clementino

04023-900 - São Paulo - SP

e-mail: gildacs@patologia.epm.br carcinomas é bastante heterogêneo, tendo sido caracterizadas algumas variáveis como fatores histológicos de prognóstico ${ }^{1}$.

A graduação histológica, a infiltração estromal linfoplasmocitária, a invasão vascular, linfática e sanguínea e a infiltração perineural são alguns dos aspectos morfológicos descritos como de importância prognóstica. Dentre estes, o grau histológico tem sido demonstrado como excelente indicador prognóstico ${ }^{2-4}$.

A avaliação dos parâmetros morfológicos de significado prognóstico tem sido realizada em espécimes de biópsia incisional ou de exérese de nódulos palpáveis. Os meios diagnósticos vêm-se 
tornando mais caros e complexos, quanto menor for a lesão suspeita ${ }^{5}$.

A punção aspirativa por agulha fina (PAAF), desde a coleta de material e subseqüente estudo citológico, tem sido considerada técnica simples, de baixo custo e com raras complicações para a paciente ${ }^{6}$. Apesar de ser método útil no diagnóstico de carcinoma de mama, existem, ainda, poucos dados a respeito da acurácia da PAAF em estabelecer fatores prognósticos, tais como: o grau histológico e nuclear, o índice mitótico, receptores hormonais, genes de supressão tumoral/ oncogene e o conteúdo de $\mathrm{DNA}^{7}$.

Alguns estudos têm mostrado que a graduação citológica nos esfregaços de carcinoma de mama correlaciona-se bem com a graduação histológica, sendo altamente reprodutive1 ${ }^{7,8-16}$. Contudo, a graduação citológica de PAAF de mama não é rotineiramente utilizada ${ }^{17}$.

Devido ao fato de terapia neoadjuvante ser utilizada como tratamento primário para carcinoma de mama, a graduação tumoral em aspirados citológicos pode ser útil nos casos de pacientes que receberão radioterapia, quimioterapia e/ou terapia hormonal como tratamento primário ${ }^{7}$. Temse, portanto, procurado graduar os tumores nos aspirados citológicos por agulha fina, para evitar possiveis erros na graduação dos espécimes cirúrgicos subseqüentes, devido a alterações histológicas relacionadas a estas terapias.

Várias classificações cito-prognósticas têm sido propostas, aplicáveis para preparados citológicos e histológicos ${ }^{8-12,18,19}$. Algumas classificações baseiam-se em critérios exclusivamente nucleares, enquanto outras associam aspectos arquiteturais e nucleares.

Os critérios nucleares mais úteis e comumente utilizados nos sistemas de graduação são a comparação do tamanho do núcleo da célula tumoral com o linfócito maduro ou hemácia, o número de mitoses, a presença e a proeminência de nucléolo e a anisonucleose ${ }^{17}$.

O presente trabalho teve como objetivos: aplicar classificações citológicas de graduação tumoral e nuclear nas PAAF e compará-las com os métodos de graduação tumoral e nuclear utilizados na histologia para identificar aquelas que mais concordam com esses graus nos respectivos preparados histológicos.

\section{Materiais e Métodos}

Foi realizado estudo retrospectivo de 132 casos de PAAF previamente diagnosticados como positivos para células neoplásicas, no período de
1985 a 1996, do arquivo do Setor de Mastologia do Departamento de Ginecologia (UNIFESP-EPM). Destes, 50 casos foram analisados e selecionados como adequados para este trabalho de acordo com os seguintes critérios: diagnóstico de carcinoma ductal invasivo confirmado posteriormente na histologia; coloração de Papanicolaou satisfatória nos esfregaços para avaliação dos critérios nucleares e citológicos; esfregaços representativos; preparados histológicos de biópsia ou ressecção cirúrgica correspondentes ao local da PAAF e fixação adequada do material histológico. As lâminas dos espécimes cirúrgicos correspondentes, coradas pela técnica de hematoxilina-eosina, do arquivo do Departamento de Patologia (UNIFESPEPM), foram reavaliadas. A graduação tanto citológica como histológica foi realizada utilizando os critérios de cada classificação.

Para avaliação da graduação tumoral foram aplicadas duas classificações citológicas que utilizam critérios arquiteturais e nucleares nos esfregaços de PAAF: sistemas de Mouriquand ${ }^{18} \mathrm{e}$ de Guilford ${ }^{20}$. Essas classificações foram comparadas com o método de graduação tumoral histológica de Scarff-Bloom-Richardson (SBR) modificado por Elston e Ellis ${ }^{3}$, utilizado para graduação dos cortes histológicos.

Para graduação nuclear foram utilizadas nos esfregaços três classificações citológicas que apresentam apenas critérios nucleares: sistema de Black modificado por Fisher et al. ${ }^{21}$ (BM), sistema de Black simplificado (BS ${ }^{10,12}$ e classificação de Hunt ${ }^{9}$. Essas classificações foram comparadas com o sistema de graduação nuclear de Black modificado por Fisher et al. ${ }^{21}$, utilizado para avaliação dos cortes histológicos.

Os esfregaços foram analisados sem o conhecimento dos subseqüentes graus tumoral e nuclear histológicos das neoplasias. Foram comparadas as diferentes classificações para graduação nuclear e tumoral nas PAAF e espécimes cirúrgicos correspondentes.

Para tumores com dupla população de células, foram considerados os escores das células mais anaplásicas.

Para análise estatística construíram-se tabelas de contingência cruzando-se cada classificação tumoral histológica com a citológica e posteriormente cada classificação nuclear citológica com a histológica. Nessas tabelas, a diagonal principal representa a concordância entre as variáveis em questão.

Para avaliar concordância entre os dois métodos, utilizou-se a medida de Kappa ${ }^{22}$, que é baseada no número de respostas concordantes, ou seja, no número de casos cujo resultado é o mesmo nos dois métodos. O Kappa corresponde à medida de 
concordância onde o valor 0 indica nenhuma concordância e o valor 1 representa total concordância. Foi usada como parâmetro para o K estatístico a graduação relativa dividida por Landis e Koch ${ }^{23}$.

O projeto de pesquisa deste trabalho foi avaliado e aprovado pelo Comitê de Ética em Pesquisa da Universidade Federal de São Paulo

\section{Resultados}

Todos os cortes histológicos e esfregaços de PAAF correspondentes foram analisados e graduados pelas diferentes classificações, de acordo com os critérios propostos.

Com a avaliação da graduação tumoral nos esfregaços utilizando-se a classificação de Mouriquand, observou-se que 25 casos (50\%) foram classificados como pouco diferenciados ou grau 3, 16 casos (32\%), como moderadamente diferenciados ou grau 2 e nove casos (18\%), como bem diferenciados ou grau 1. Utilizando-se o sistema de Guilford observou-se que dentre os 50 casos, 24 (48\%) casos foram grau 3, 15 (30\%) casos foram grau 2 e $11(22 \%)$ casos foram grau 1 .

A avaliação do grau nuclear nos esfregaços utilizando-se o sistema de Black modificado por Fisher et al. ${ }^{21}$ classificou trinta e um casos (62\%) como pouco diferenciados (grau 3), 17 casos (34\%) como moderadamente diferenciados (grau 2) e dois casos (4\%) como bem diferenciados (grau 1). Usando-se o sistema de BS verificou-se que 26 casos $(52 \%)$ foram considerados de alto grau e 24 (48\%) de baixo grau. Com o sistema de Hunt ${ }^{9}$, dos 50 casos estudados e graduados, 15 (30\%) foram de baixo grau e $35(70 \%)$ foram de alto grau.

A avaliação histológica do grau tumoral utilizando-se o sistema SBR modificado por Elston e Ellis ${ }^{3}$ revelou: para o grau 3 ou pouco diferenciado foram encontrados 24 casos (48\%); para grau 2 ou moderadamente diferenciado, 21 casos (42\%), e para grau 1 ou bem diferenciado, cinco casos (10\%). Quando a relação era feita com a classificação em que se utilizavam duas categorias (baixo e alto graus - BS), o grau 2 dividia-se em grau 1 ou 3. Para isto, todos os casos de grau 2 foram revistos: aqueles em que a soma dos escores era sete, foram considerados grau 3, e os que somaram seis, grau 1. Onze casos foram reclassificados como baixo grau e 35 casos como alto grau.

A avaliação histológica do grau nuclear utilizando-se o sistema de Black modificado por Fisher et al. ${ }^{21}$ revelou: para grau 3 ou pouco diferenciado foram encontrados 32 casos (64\%); para grau 2 ou moderadamente diferenciado, 15 casos (30\%), e para grau 1 ou bem diferenciado, três casos (6\%). Quando a relação de concordância era feita com classificações baseadas em duas categorias (BS e Hunt ${ }^{9}$ ), o grau 2 foi reavaliado para separar aqueles casos que poderiam ser considerados grau 1 ou 3 . Os casos que apresentavam mitose e nucléolo proeminentes foram considerados grau 3, e aqueles em que estes critérios não estavam evidentes foram classificados como grau 1. Com esta divisão, foram chamados de alto (41 casos) e baixo (nove casos) graus.

O valor de kappa para os sistemas de graduação de Mouriquand e de Guilford foram, respectivamente, $\mathrm{K}=0,210$ (Tabela1) e $\mathrm{K}=0,260$ (Tabela 2). Dentre esses sistemas de graduação, que utilizam critérios tumorais, o sistema de Guilford apresentou maior concordância com o sistema de SBR modificado (Tabela 3).

Tabela 1 - Condordância entre os sistemas de graduação de SBR* modificado e o de Mouriquand.

\begin{tabular}{ccccc}
$\begin{array}{c}\text { Mouriquand } \\
\text { Graus }\end{array}$ & \multicolumn{4}{c}{$\begin{array}{c}\text { SBR modificado } \\
\text { Graus }\end{array}$} \\
\hline & 1 & 2 & 3 & Total \\
1 & 2 & 4 & 3 & 9 \\
2 & 3 & 8 & 5 & 16 \\
3 & 0 & 9 & 16 & 25 \\
Total & 5 & 21 & 24 & 50 \\
\hline
\end{tabular}

*Scarff, Bloom e Richardson

Kappa $=0,210$ (erro padrão $=0,109$ ) $p=0,047$

Tabela 2 - Concordância entre os sistemas de graduação de SBR* modificado e o de Guilford.

\begin{tabular}{|c|c|c|c|c|}
\hline $\begin{array}{l}\text { Guilford } \\
\text { Graus }\end{array}$ & & $\begin{array}{l}\text { nodi } \\
\text { Grau }\end{array}$ & & \\
\hline & 1 & 2 & 3 & Total \\
\hline 1 & 2 & 2 & 3 & 11 \\
\hline 2 & 3 & 5 & 3 & 15 \\
\hline 3 & 0 & 8 & 16 & 24 \\
\hline Total & 5 & 21 & 24 & 50 \\
\hline
\end{tabular}

*Scarff, Bloom e Richardson

Kappa $=0,260$ (erro padrão $=0,107$ )

$p=0,011$

Tabela 3 - Comparações do sistema de graduação de SBR* modificado com os sistemas de Mouriquand e de Guilford.

\begin{tabular}{lccc}
\hline & $\begin{array}{c}\text { Medida de } \\
\text { Kappa }\end{array}$ & Erro padrão & $\begin{array}{c}\text { Nível } \\
\text { descritivo }\end{array}$ \\
\hline Mouriquand & 0,210 & 0,109 & 0,047 \\
Guilford & 0,260 & 0,107 & 0,011 \\
\hline
\end{tabular}

*Scarff, Bloom e Richardson

Dentre os sistemas de graduação nuclear, o sistema de BM $(\mathrm{K}=0,358)$ apresentou maior concordância com o sistema de BM para histologia. Os valores de Kappa para os sistemas de BS e de Hunt $^{9}$ foram, respectivamente, $\mathrm{K}=0,302$ e $\mathrm{K}=0,140$ (Tabela 4). 
Tabela 4 - Comparações do sistema de graduação de BM para histologia com os sistemas de BM para citologia, de BS e de Hunt.

\begin{tabular}{lccc}
\hline & $\begin{array}{c}\text { Medida de } \\
\text { Kappa }\end{array}$ & Erro padrão & $\begin{array}{c}\text { Nível } \\
\text { descritivo }\end{array}$ \\
\hline BM & 0,358 & 0,123 & 0,003 \\
BS & 0,302 & 0,108 & 0,007 \\
Hunt & 0,140 & 0,144 & 0,296 \\
\hline
\end{tabular}

BM: Black modificado

BS: Black simplificado

Apesar de os valores de Kappa terem sido significantes, nos sistemas de graduação de BM, de BS, de Guilford e de Mouriquand, os níveis descritivos, segundo Landis e Koch ${ }^{23}$, foram considerados regulares.

\section{Discussão}

Neste estudo não foi avaliada a influência dos critérios separadamente na graduação final, no entanto, ficou evidente que aqueles sistemas que utilizam critérios nucleares apresentaram maior concordância.

Dentre os métodos de graduação nuclear citológica (sistemas de BM, de BS e de Hunt ${ }^{9}$ ), a classificação de BM apresentou maior concordância com o encontrado por outros autores ${ }^{7,24}$. O sistema de BS que aplica apenas dois graus também correlacionou-se significativamente com o grau histológico.

Os sistemas que utilizam duas categorias são considerados simples e práticos, obtendo melhor reprodutibilidade intra e interobservador, além de melhor discriminação terapêutica e prognóstica ${ }^{9,17}$.

Nesse estudo, os dois sistemas apresentaram maior concordância. No entanto, o sistema com três categorias (BM) mostrou-se mais concordante que o de duas (BS). Foi observado que todos os casos considerados grau 2 no sistema de BM foram baixo grau pelo método de BS. Taxas semelhantes foram encontradas, também, por outros autores ${ }^{17}$. Portanto, a aplicação de um sistema com duas categorias induz o grau 2 a tornar-se baixo grau, e isto pode não reproduzir o verdadeiro grau. Outro trabalho demonstrou que a concordância cito-histológica entre os sistemas de Hunt ${ }^{9}$, com duas categorias, e o SBR modificado, com três categorias, também revelou maior porcentagem de baixo grau (dez casos) quando foi grau 2 na histologia (14 casos) $)^{24}$.

Apesar de ter sido baseado no sistema de SBR modificado, o sistema de Hunt ${ }^{9}$ avalia apenas diâmetro e pleomorfismo nuclear, nucléolo múltiplo e facilmente visivel. Sendo assim, esta classifi- cação foi incluída na comparação com o sistema de graduação de BM, que apresenta, exclusivamente, critérios nucleares.

Dos métodos que aplicam critérios nucleares, os sistemas de BM e BS apresentaram maior concordância em relação ao sistema de Hunt ${ }^{9}$. Ambos os sistemas incluem anisonucleose, contagem mitótica e tamanho do núcleo em seus critérios. O grau nuclear citológico, avaliado pelo método de BM, mostrou-se reprodutível, correlacionando-se precisamente com o grau nuclear histológico ${ }^{19,25}$.

Tem sido demonstrado que aspectos nucleares mostram maior correlação entre material histológico e citológico, especialmente pleomorfismo nuclear $8,9,11,16,26$. A presença de nucléolo tem sido outro aspecto concordante ${ }^{9,20,26}$, entretanto, outro estudo não encontrou correlação ${ }^{8}$. Esta discrepância tem sido explicada possivelmente por diferenças nos métodos de fixação e coloração usados nos diferentes trabalhos ${ }^{9}$. Recentemente, concluiu-se que diferenças sistemáticas entre patologistas em avaliar o pleomorfismo nuclear de cortes histológicos de carcinomas de mama potencialmente contribuem para diferenças na graduação final ${ }^{27}$. Em um outro estudo citológico, a dissociação e o nucléolo foram os fatores mais influentes na graduação de carcinoma de mama ${ }^{20}$.

O tamanho do núcleo tem sido outro elemento em várias classificações citológicas. Entretanto, alguns trabalhos que avaliam este parâmetro têm demonstrado sobreposição significativa entre carcinomas de baixo grau e condições benignas ${ }^{8,28}$. Outro ponto considerado tem sido a influência do método de fixação, do tempo de exposição ao ar e da coloração na avaliação do tamanho nuclear. A acurácia do sistema de graduação pode ser prejudicada pela falta de uniformidade na preparação do esfregaço, principalmente quando a comparação do diâmetro dos núcleos malignos com a hemácia mais próxima é utilizada como critério de graduação ${ }^{17}$.

Os esfregaços corados tanto pela técnica de Papanicolaou como pelas técnicas de Romanowski podem ser utilizados para graduação nuclear citológica. Todavia, o material seco ao ar propicia maior acentuação das variações dos diâmetros nucleares, ao passo que os nucléolos mostram-se mais inconspícuos ${ }^{19}$. Outros preferem a coloração de Papanicolaou por considerá-la ideal, devido ao detalhe nuclear ótico produzido pela técnica ${ }^{29}$.

Outro estudo encontrou que o grau nuclear e o padrão do esfregaço definido por porcentagem de células em lençóis, em agrupamentos e isoladas estavam associados com a presença de metástase à distância. Os autores não correlacionaram seus resultados com grau histológico ${ }^{30}$. 
A freqüência de figuras mitóticas tem sido avaliada em diferentes sistemas de graduação; entretanto, disparidades nos graus citológico e histológico em alguns casos têm sido atribuídas, principalmente, a dificuldades em se detectarem mitoses ou túbulos na citologia ${ }^{11,15,25}$.

Dentre os métodos de graduação tumoral citológica (sistemas de Mouriquand e de Guilford), o sistema de Guilford apresentou maior concordância. Todo sistema de graduação apresenta certo grau de subjetividade com análise de vários fatores. Aqueles que aplicam apenas poucos parâmetros dificultam a análise, por restringir as variáveis. $\mathrm{O}$ sistema de Guilford baseia-se em seis parâmetros e três variáveis. Na padronização de critérios para PAAF de mama, desenvolvidos por grupo multidisciplinar de especialistas, recomenda-se a utilização desse sistema na citologia quando, para a histologia, for aplicado o sistema de SBR modificado ${ }^{31}$.

O sistema de graduação de Mouriquand não utiliza o critério de tamanho e diâmetro nuclear, observado nos demais sistemas do presente estudo. A pouca significância deste método pode ser atribuída a falta desses critérios. Utilizando esse mesmo sistema, outro trabalho concluiu que a importância prognóstica dessa graduação citológica é baixa e dependente de outros fatores prognósticos clássicos, não tendo valor prático ${ }^{32}$.

Os resultados da graduação histológica pela classificação de SBR modificada revelaram maior número de casos grau 3 (24 casos - 48\%) e menor freqüência do grau 1 (cinco casos - 10\%), sendo semelhantes aos encontrados por outros auto$\mathrm{res}^{3,24}$. A maior concordância nos casos grau $3 \mathrm{em}$ todas as classificações pode ser justificada pelo maior número de casos deste grau e por haver maior facilidade em identificar seus critérios em relação aos demais graus. Outros trabalhos, também, relatam taxas semelhantes ${ }^{7,11,24}$.

Têm-se descrito diferentes índices de reprodutibilidade para graduação histológica, com taxa de concordância variando de moderada a substancial $^{33}$. Concordância completa foi observada em $73,5 \%$ de tumores fixados em formol tamponado ${ }^{34}$. Estudo recente demonstrou a utilidade de métodos histopatológicos quantitativos com a avaliação prognóstica de sistema de graduação morfométrico ${ }^{35}$.

A variabilidade dos resultados observados na graduação dos esfregaços de PAAF pode também refletir variações similares àquelas descritas para graduação histológica.

Os sistemas de graduação apresentaram regular significância estatística. Aqueles que aplicam, exclusivamente, critérios nucleares apresentaram maior concordância do que aqueles que combinam parâmetros nucleares e arquiteturais. As classificações de escores com mais categorias e critérios morfológicos a serem avaliados possibilitaram menor margem de erro.

Os componentes dos sistemas de graduação são relativamente simples de serem aplicados, requerendo um mínimo de equipamento especializado. No entanto, a variabilidade dos achados sugere a necessidade de sistemas com parâmetros mais objetivos e precisos para sua utilização na rotina da citopatologia.

\section{ABSTRACT}

Purpose: to assess the concordance of cytological tumoral and nuclear grading systems on fine needle aspiration biopsies of breast carcinoma with histological specimens and compare them to identify the best results.

Methods: cytohistological agreement was evaluated in a retrospective study of 50 cases of fine needle aspiration biopsies of histologically confirmed invasive ductal carcinoma of the breast, with 5 grading systems being applied for comparative purposes.

The classifications were divided according to criteria of tumoral grading (nuclear and architectural criteria Mouriquand and Guilford systems) and nuclear criteria (Black modified by Fisher (BM), simplified Black system (SB) and Hunt system). The grading systems used for histological analysis were those of Scarff-Bloom-Richardson modified by Elston (SBR modified) for tumor evaluation and the BM systems for nuclear evaluation.

Results: the cytological grading systems that showed best agreement were BM and SB based on nuclear criteria (anisonucleosis, size, mitosis, and chromatin). Among the cytological grading systems based on nuclear and architectural criteria (combined), Guilford's classification showed the best agreement, possibly due to the larger number of variables used, which permitted a smaller margin of error. Conclusion: the methods evaluated in the present study can be considered reasonable as cytological grading systems.

KEYWORDS: Breast carcinoma. Nuclear grading. Histological grading. Fine needle aspiration

\section{Referências}

1. Rosen PP. The pathological classification of human mammary carcinoma: past, present and future. Ann Clin Lab Sci 1979; 9:144-56.

2. Contesso G, Mouriesse H, Friedman S, Genin J, Sarrazin D, Rouesse J. The importance of histologic grade in long-term prognosis of breast cancer: a study of 1,010 patients, uniformly treated at the Institut Gustave-Roussy. J Clin Oncol 1987; 5:1378-86.

3. Elston CW, Ellis IO. Pathological prognostic factors in breast cancer. I. The value of histological grade in breast cancer: experience from a large study with long-term follow-up. Histopathology 1991; 19:403-10. 
4. Henson DE, Ries L, Freedman LS, Carriaga M. Relationship among outcome, stage of disease, and histologic grade for 22,616 cases of breast cancer: the basis for a prognostic index. Cancer 1991; 68:2142-9.

5. Layfield LJ, Chrischilles EA, Cohen MB, Bottles K. The palpable breast nodule: a cost-effectiveness analysis of alternate diagnostic approaches. Cancer 1993; 72:1642-51.

6. Koss LG. The palpable breast nodule: a costeffectiveness analysis of alternate diagnostic approaches: the role of the needle aspiration biopsy. Cancer 1993; 72:1499-502.

7. Zoppi JA, Pellicer EM, Sundblad AS. Cytohistologic correlation of nuclear grade in breast carcinoma. Acta Cytol 1997; 41:701-4.

8. Thomas JS, Mallon EA, George WD. Semiquantitative analysis of fine needle aspirates from benign and malignant breast lesions. J Clin Pathol 1989; 42:28-34.

9. Hunt CM, Ellis IO, Elston CW, Locker A, Pearson D, Blamey RW. Cytological grading of breast carcinomaa feasible proposition? Cytopathology 1990; 1:287-95.

10.Cajulis R, Sneige N, El-Naggar A. Cytologic nuclear grading of fine needle aspirates of breast carcinoma: concordance with corresponding histologic grading and flow cytometric data. Mod Pathol 1990; 3:14A.

11.Ducatman BS, Emery ST, Wang HH. Correlation of histologic grade of breast carcinoma with cytologic features on fine-needle aspiration of the breast. Mod Pathol 1993; 6:539-43.

12.Cajulis RS, Hessel RG, Hwang S, Haines K, FriasHidvegi D, O'Gorman M. Simplified nuclear grading of fine-needle aspirates of breast carcinoma: concordance with corresponding histologic nuclear grading and flow cytometric data. Diagn Cytopathol 1994; 11:124-30.

13. Moroz K, Lipscomb J, Vial LJ, Dhurandhar N. Cytologic nuclear grade of malignant breast aspirates as a predictor of histologic grade: light microscopy and image analysis characteristics. Acta Cytol 1997; 41:1107-11.

14.Briffod M, Le Doussal V, Spyratos F. Cytologic nuclear grading of fine needle cytopunctures of breast carcinoma. Comparison with histologic nuclear grading and image cytometric data. Anal Quant Cytol Histol 1997; 19:114-22.

15. Howell LP, Gandour-Edwards R, O'Sullivan D. Application of the Scarff-Bloom-Richardson tumor grading system to fine-needle aspirates of the breast. Am J Clin Pathol 1994; 101:262-5.

16.Kalogeraki A, Tamiolakis D, Kozoni V, et al. Nuclear grading in invasive ductal breast carcinomas. Cancer Detect Prev 2000; 24:224-7.

17.Cajulis RS, Hessel RG, Frias-Hidvegi D, Yu GH. Cytologic grading of fine needle aspirates of breast carcinoma by private practice pathologists. Acta Cytol 1997; 41:313-20.

18. Mouriquand J, Pasquier D. Fine needle aspiration of breast carcinoma: a preliminary cytoprognostic study. Acta Cytol 1980; 24:153-9.
19.Dabbs DJ. Role of nuclear grading of breast carcinomas in fine needle aspiration specimens. Acta Cytol 1993; 37:361-6.

20.Robinson IA, McKee G, Nicholson A, et al. Prognostic value of cytological grading of fine-needle aspirates from breast carcinomas. Lancet 1994; 343:947-9.

21.Fisher ER, Redmond C, Fisher B. Histologic grading of breast cancer. Pathol Annu 1980; 15:239-51.

22.Agresti A. Categorical data analysis. $1^{\text {st }}$ ed. New York: Wiley; 1990.

23.Landis JR, Koch GG. The measurement of observer agreement for categorical data. Biometrics 1977; 33:159-74.

24. New NE, Howat AJ. Nuclear grading of breast carcinoma. Acta Cytol 1994; 38:969-70.

25.Dabbs DJ, Silverman JF. Prognostic factors from the fine-needle aspirate: breast carcinoma nuclear grade. Diagn Cytopathol 1994; 10:203-8.

26. Mouriquand J, Gozlan-Fior M, Villemain D, et al. Value of cytoprognostic classification in breast carcinomas. J Clin Pathol 1986; 39:489-96.

27.Dunne B, Going JJ. Scoring nuclear pleomorphism in breast cancer. Histopathology 2001; 39:259-65.

28.Zajdela A, De LaRiva LS, Ghossein NA. The relation of prognosis to the nuclear diameter of breast cancer cells obtained by cytologic aspiration. Acta Cytol 1979; 23:75-80.

29. Sneige N. Current issues in fine-needle aspiration of the breast: cytologic features of in situ lobular and ductal carcinomas and clinical implications of nuclear grading. Cytopathol Annu 1992; 7:155-71.

30.Layfield LJ, Robert ME, Cramer H, Giuliano A. Aspiration biopsy smear pattern as a predictor of biologic behavior in adenocarcinoma of the breast. Acta Cytol 1992; 36:208-14.

31. The uniform approach to breast fine-needle aspiration biopsy. National Cancer Institute Fine-Needle Aspiration of Breast Workshop Subcommittees. Diagn Cytopathol 1997; 16:295-311.

32. Ciatto S, Bonardi R, Herd-Smith A, Cariaggi P, Confortini M, Bulgaresi P. Prognostic value of breast cancer cytologic grading: a retrospective study of 213 cases. Diagn Cytopathol 1993; 9:160-3.

33. Frierson HF Jr, Wolber RA, Berean KW, et al. Interobserver reproducibility of the Nottingham modification of the Bloom and Richardson histologic grading scheme for infiltrating ductal carcinoma. Am J Clin Pathol 1995; 103:195-8.

34.Robbins P, Pinder S, de Klerk N, et al. Histological grading of breast carcinomas: a study of interobserver agreement. Hum Pathol 1995; 26:873-9.

35.Kronqvist P, Kuopio T, Jalava P, Collan Y. Morphometrical malignancy grading is a valuable prognostic factor in invasive ductal breast cancer. Br J Cancer 2002; 87:1275-80.

Recebido em: 25/2/2003

Aceito após modificações em: 10/3/2003 